\title{
A CONSTRUCTION FOR PSEUDOCOMPLEMENTED SEMILATTICES AND TWO APPLICATIONS
}

\author{
M. E. ADAMS AND MATTHEW GOULD \\ (Communicated by Louis J. Ratliff, Jr.)
}

\begin{abstract}
A method is given by which pseudocomplemented semilattices can be constructed from graphs. Two consequences of the method are obtained, namely: there exist continuum-many quasivarieties of pseudocomplemented semilattices; for any non-zero cardinal $\kappa$, there exist $\kappa$ pairwise non-isomorphic pseudocomplemented semilattices with isomorphic endomorphism monoids.
\end{abstract}

\section{INTRODUCTION}

A pseudocomplemented semilattice is an algebra $(S ; \wedge, *, 0,1)$ of type $(2,1,0,0)$ consisting of a semilattice $(S ; \wedge)$ with a least element 0 , a greatest element 1 , and a pseudocomplementation operation ${ }^{*}$ such that, for $x, y \in S$, $x \wedge y=0$ if and only if $y \leq x^{*}$.

We shall exhibit a method by which pseudocomplemented semilattices can be constructed from graphs. By applying this construction, the following results will be established.

Theorem 1. There exist $2^{\aleph_{0}}$ quasivarieties of pseudocomplemented semilattices.

Theorem 2. Given a non-zero cardinal $\kappa$, there exists a family of pseudocomplemented semilattices $\left(S_{i}: i \in I\right)$ satisfying

(i) $S_{i} \not S_{j}$ for distinct $i, j \in I$;

(ii) $\operatorname{End}\left(S_{i}\right) \cong \operatorname{End}\left(S_{j}\right)$ for all $i, j \in I$;

(iii) if $\kappa$ is infinite, then $|I|=2^{\kappa}$ and $\left|S_{i}\right|=\kappa$ for all $i \in I$;

(iv) if $\kappa$ is finite, then $|I|=\kappa$ and each $S_{i}$ is finite.

In Theorem 2, and throughout this paper, the notation $\operatorname{End}(S)$ denotes the monoid (semigroup with identity) of all endomorphisms of $S$ with composition as multiplication.

It should be noted that Theorem 1 stands in striking contrast to the fact that there are only two non-trivial varieties of pseudocomplemented semilattices,

Received by the editors November 28, 1988. $08 \mathrm{C} 15$

1980 Mathematics Subject Classification (1985 Revision). Primary 06A12; Secondary 08A35, 
proved by Jones [7] (cf. Sankappanavar [10]). That not every quasivariety of pseudocomplemented semilattices is necessarily a variety had already been observed by Sankappanavar (unpublished) and, independently, Schmid [12].

Both theorems have precise analogues for pseudocomplemented distributive lattices, i.e., algebras $\left(L ; \vee, \wedge,{ }^{*}, 0,1\right)$ such that $(L ; \vee, \wedge)$ is a distributive lattice and $\left(L ; \wedge,{ }^{*}, 0,1\right)$ is a pseudocomplemented semilattice. The analogue of Theorem 1 is given in [1] and, independently, Wronski [13]; the analogue of Theorem 2 was proved in [2]. For Boolean algebras, both theorems fail spectacularly: there is only one non-trivial quasivariety, and Boolean algebras having isomorphic endomorphism monoids are isomorphic (Magill [8], Maxson [9], and Schein [11]).

The reader in need of background material concerning pseudocomplemented semilattices and related topics is directed to Grätzer [3]. We shall use the following notations. If $S$ is a pseudocomplemented semilattice, $S^{*}$ denotes the skeleton of $S$, that is, $S^{*}=\left\{x^{*}: x \in S\right\}$. The Glivenko congruence on $S$, denoted $\Gamma_{S}$, is defined by $\Gamma_{S}=\left\{(x, y) \in S \times S: x^{*}=y^{*}\right\}$. The Glivenko endomorphism of $S$, denoted $\gamma_{S}$, is defined by $\gamma_{S}(x)=x^{* *}$ for all $x \in S$. Because $x^{*}=x^{* * *}$ for all $x \in S$, it follows that $\gamma_{S}$ is the identity on $S^{*}$ and that $\Gamma_{S}$ is the congruence induced by $\gamma_{S}$, i.e., $\Gamma_{S}=\left\{(x, y) \in S \times S: \gamma_{S}(x)=\gamma_{S}(y)\right\}$. Since $S^{*}$ is a Boolean lattice where $x^{*} \vee y^{*}=\left(x^{* *} \wedge y^{* *}\right)^{*}$ for any $x, y \in S$, it follows that if $\varphi: S \rightarrow T$ is a homomorphism to a pseudocomplemented semilattice $T$, then $\varphi \mid S^{*}: S^{*} \rightarrow T^{*}$ is a Boolean homomorphism.

\section{THE CONSTRUCTION}

The immediate goal of this section is to define a pseudocomplemented semilattice $S_{G}$ for every graph $G$.

For a graph $G=(V, E)$ (i.e., a set $V$ together with a set $E$ of two-element subsets of $V$ ), let $B_{G}$ denote the Boolean lattice of finite/co-finite subsets of $V$ ordered by inclusion. Further, let

$$
S_{G}=\left(B_{G} \times 2\right) \backslash(\{(\varnothing, 1),(V, 0)\} \cup\{(a, 0):|a|=1 \text { or } a \in E\})
$$

where 2 denotes the two-element chain $\{0,1\}$. Let $\leq$ denote the usual ordering on $B_{G} \times 2$ and define a relation $\leqq$ on $S_{G}$ by $(a, i) \leqq(b, j)$ iff either $(a, i) \leq(b, j)$ in $B_{G} \times 2$,

$$
\begin{aligned}
& \text { or } i=1, j=0, a \leq b, \text { and }|a|=1, \\
& \text { or } i=1, j=0, a \leq b, \text { and } a \in E .
\end{aligned}
$$

It must be shown that $\left(S_{G}, \leqq\right)$ is indeed a pseudocomplemented semilattice.

Lemma 1. $\leqq$ is an order relation.

Proof. Since $\leq$ is reflexive, so too is $\leqq$.

To see that $\leqq$ is anti-symmetric, first note that the only new pairs added to $\leq$ are of the form $(a, 1) \leqq(b, 0)$, whence we may suppose that

$$
(a, 1) \leqq(b, 0) \text { and }(b, 0) \leqq(a, 1) \text {. }
$$


It follows that $a=b$. Further, by the first inequality, either $|a|=1$ or $a \in E$ which is absurd, since in neither case is $(a, 0)$ an element of $S_{G}$.

Finally, to see that $\leqq$ is transitive, we need only consider cases in which one inequality is of the form $(a, 1) \leqq(b, 0)$. Thus, either

$$
\begin{aligned}
& (c, i) \leqq(a, 1) \text { and }(a, 1) \leqq(b, 0) \quad \text { or } \\
& (a, 1) \leqq(b, 0) \text { and }(b, 0) \leqq(c, i)
\end{aligned}
$$

for $i=0,1$. In either case, $a \leq b$ and $|a|=1$ or $a \in E$. If, in the former case, $c=\varnothing$, then $i=0$ and $(c, 0) \leqq(b, 0)$. Otherwise, since $c \leq a \leq b$, $|c|=1$ or $c=a \in E$. No matter which, $i=1$ and $(c, 1) \leqq(b, 0)$. In the latter case $a \leq c$ and, hence, $(a, 1) \leqq(c, i)$.

Lemma 2. $S_{G}$ is a semilattice where, for $(a, i),(b, j) \in S_{G}$,

$$
(a, i) \wedge(b, j)= \begin{cases}(\varnothing, 0) & \text { if } a \wedge b=\varnothing, \\ (a \wedge b, 1) & \text { if }|a \wedge b|=1 \text { or } a \wedge b \in E, \\ (a \wedge b, i \wedge j) & \text { otherwise. }\end{cases}
$$

Proof. Suppose $(a, i),(b, j) \in S_{G}$ and $(c, k)$ is a common lower bound. In particular, $c \leq a \wedge b$. We consider the various possibilities.

If $a \wedge b=\varnothing$, then $(\varnothing, 0)$ is a common lower bound. Since $c=\varnothing$ in this case, $(\varnothing, 0)$ is the only lower bound.

If $|a \wedge b|=1$ or $a \wedge b \in E$, then $(a \wedge b, 1)$ is a common lower bound. Moreover, it is the greatest since $c \leq a \wedge b$ implies that $(c, k) \leqq(a \wedge b, 1)$ for any $k$.

Finally, suppose $|a \wedge b| \geq 2$ and $a \wedge b \notin E$. If $(a \wedge b, i \wedge j)=(V, 0)$, then either $(a, i)$ or $(b, j)=(V, 0)$ which is absurd. Thus, $(a \wedge b, i \wedge j) \in S_{G}$ by hypothesis. Clearly, $(a \wedge b, i \wedge j)$ is a common lower bound; it is to be seen that it is the greatest. If $k=0$, then $(c, 0) \leqq(a \wedge b, i \wedge j)$ automatically. Suppose, on the other hand, that $k=1$. For $i=j=1,(c, 1) \leqq(a \wedge b, 1)$. Otherwise, say, $i=0$. Thus $(c, 1) \leqq(a, 0)$ and so $|c|=1$ or $c \in E$. Either way, $(c, 1) \leqq(a \wedge b, i \wedge j)$.

Lemma 3. $S_{G}$ is a pseudocomplemented semilattice where $(V, 1)^{*}=(\varnothing, 0)$ and, for $(V, 1) \neq(a, i) \in S_{G},(a, i)^{*}=\left(a^{*}, 1\right)$.

Proof. Obviously, $(V, 1)^{*}=(\varnothing, 0)$. For $(V, 1) \neq(a, i) \in S_{G}, a \neq V$. Thus, $a^{*} \neq \varnothing$ and, hence, $\left(a^{*}, 1\right) \in S_{G}$. By Lemma $2,(a, i) \wedge\left(a^{*}, 1\right)=(\varnothing, 0)$. Furthermore, if $(a, i) \wedge(b, j)=(\varnothing, 0)$, then $a \wedge b=\varnothing$. In particular, $b \leq a^{*}$ and $(b, j) \leqq\left(a^{*}, 1\right)$.

The remainder of this section establishes the properties of pseudocomplemented semilattices of the form $S_{G}$ that will be required in the proofs of Theorems 1 and 2.

Let $G=(V, E)$ and $H=(W, F)$ be two graphs such that $|V| \geq 5$ and, for every $x \in V, x \in e$ for some $e \in E$. Further, let $\varphi: S_{G} \rightarrow S_{H}$ be a homomorphism and let $\theta$ denote the congruence on $S_{G}$ induced by $\varphi$. 
Lemma 4. If, for every co-atom a of $B_{G},(a, 0) \equiv(a, 1)(\theta)$, then $\theta \supseteq \Gamma_{S_{G}}$.

Proof. For any $(b, 0),(b, 1) \in S_{G}$, there exists a co-atom $a \in B_{G}$ such that $a \geq b$. Since $(b, 0) \in S_{G},|b| \neq 1$ and $b \notin E$ and, since $(b, 1) \in S_{G}, b \neq \varnothing$. Thus, by Lemma $2,(a, 0) \wedge(b, 1)=(b, 0)$ and $(a, 1) \wedge(b, 1)=(b, 1)$. By hypothesis, it follows that $(b, 0) \equiv(b, 1)(\theta)$.

Lemma 5. If $\theta \nsupseteq \Gamma_{S_{G}}$, then $\varphi$ is one-to-one on $S_{G}^{*}$.

Proof. Suppose $\varphi$ is not one-to-one on $S_{G}^{*}$. Then, since $\varphi \mid S_{G}^{*}: S_{G}^{*} \rightarrow S_{H}^{*}$ is a Boolean homomorphism, $\varphi(a, 1)=\varphi(V, 1)=(W, 1)$ for some co-atom $a \in B_{G}$. Thus, by Lemma 3, $(\varphi(a, 0))^{*}=\varphi\left((a, 0)^{*}\right)=\varphi\left(a^{*}, 1\right)=\varphi\left((a, 1)^{*}\right)=$ $(\varphi(a, 1))^{*}=(W, 1)^{*}=(\varnothing, 0)$. It follows that $\varphi(a, 0)=(W, 1)$ and, hence, $(a, 0) \equiv(V, 1)(\theta)$. Let $b$ be any other co-atom of $B_{G}$. By Lemma 4 , it is sufficient to show $(b, 0) \equiv(b, 1)(\theta)$. Since $|V| \geq 5,|a \wedge b| \geq 3$. Thus, by Lemma 2, $(a \wedge b, 0)=(a, 0) \wedge(b, 1) \equiv(V, 1) \wedge(b, 1)=(b, 1)$. It follows that $(b, 0) \equiv(b, 1)(\theta)$ since $(a \wedge b, 0) \leqq(b, 0) \leqq(b, 1)$.

Lemma 6. If $\theta \nsupseteq \Gamma_{S_{G}}$, then, for $\varnothing \neq a \in B_{G}, \varphi(a, 1)=(r, 1)$ for some $r \in B_{H}$. Furthermore,

(i) if $|a|=1$, then $|r|=1$;

(ii) if $a \in E$, then $r \in F$; and

(iii) if $(a, 0) \equiv(a, 1)(\theta)$, then $|a|=2$ and $r \in F$.

Proof. By Lemma $5, \varphi$ is one-to-one on $S_{G}^{*}$. Further, since $\varphi$. $S_{G}^{*}: S_{G}^{*} \rightarrow S_{H}^{*}$, it follows that, for $\varnothing \neq a \in B_{G}, \varphi(a, 1)=(r, 1)$ for some $r \in B_{H}$.

With (iii) in mind, suppose $(a, 0) \equiv(a, 1)(\theta)$ for some $|a| \geq 3$. Then there exists a co-atom $b \in B_{G}$ such that $b \geq a$. Since $\varphi$ is order-preserving, $s \geq r$ where $\varphi(b, 1)=(s, 1)$. By hypothesis, $(a, 1) \wedge(b, 0)=(a, 0)$ and, hence, $(r, 1) \wedge \varphi(b, 0)=(r, 1)$; in particular, $\varphi(b, 0) \geqq(r, 1)$. However, since $\varphi$ preserves ${ }^{*}, \varphi(b, 0) \in\{(s, 0),(s, 1)\}$. But, because $\varphi$ is one-to-one on $S_{G}^{*},|r| \geq 3$ and, hence, $(s, 0) \geq(r, 1)$. It follows that $\varphi(b, 0)=(s, 1)$ and, consequently, that $(b, 0) \equiv(b, 1)(\theta)$. We claim that, for any co-atom $c \in B_{G}$, $(c, 0) \equiv(c, 1)(\theta)$. To see this observe that, since $|b \wedge c| \geq 3,(b \wedge c, 0)=$ $(b, 0) \wedge(b \wedge c, 1) \equiv(b, 1) \wedge(b \wedge c, 1)=(b \wedge c, 1)$. Hence, $\varphi(b \wedge c, 1)=\varphi(b \wedge c, 0)=$ $\varphi((c, 0) \wedge(b \wedge c, 1))=\varphi(c, 0) \wedge \varphi(b \wedge c, 1)$; that is, $\varphi(c, 0) \geqq \varphi(b \wedge c, 1)$. If $\varphi(c, 1)=(t, 1)$, then, since $\varphi$ preserves ${ }^{*}, \varphi(c, 0) \in\{(t, 0),(t, 1)\}$. But, if $\varphi(b \wedge c, 1)=(u, 1)$, then $|u| \geq 3$ since $\varphi$ is one-to-one on $S_{G}^{*}$. It follows that $(t, 0) \nsupseteq(u, 1)$ and, hence, $\varphi(c, 0)=(t, 1)$. Thus, $(c, 0) \equiv(c, 1)(\theta)$ for every co-atom $c \in B_{G}$. By Lemma 4 , this is absurd. Thus, we conclude that, for any $a \in B_{G}$, if $|a| \geq 3$, then $(a, 0) \not \equiv(a, 1)(\theta)$.

Suppose now that $|a|=2$. Set $b=a \cup\{x\}$ for some $x \in V \backslash a$. Since $\varphi$ is one-to-one on $S_{G}^{*},|s|>|r| \geq 2$ where $\varphi(a, 1)=(r, 1)$ and $\varphi(b, 1)=(s, 1)$. Thus, as shown above, $(b, 0) \not \equiv(b, 1)(\theta)$ and, in particular, $\varphi(b, 0)=(s, 0)$. To prove (ii), suppose $a \in E$. Then $(a, 1) \leqq(b, 0)$ and, hence, $(r, 1) \leqq(s, 0)$ which, since $|r| \geq 2$, implies $r \in F$. Thus, (ii) is seen to hold. To prove 
(iii), suppose $a \notin E$. For $r \notin F$, since $(a, 0)=(a, 1) \wedge(b, 0), \varphi(a, 0)=$ $(r, 1) \wedge(s, 0)=(r, 0)$ and, hence, $(a, 0) \not \equiv(a, 1)(\theta)$ which verifies (iii).

Since $\varphi$ is one-to-one on $S_{G}^{*}$ and $V \subseteq \cup E$, (i) is an immediate consequence of (ii).

Proposition 1. If $\theta \nsupseteq \Gamma_{S_{G}}$, then the following hold:

(i) for $x \in V, \varphi(\{x\}, 1)=(\{\psi(x)\}, 1)$ defines a one-to-one compatible mapping $\psi: G \rightarrow H$ (a mapping is compatible if $\{\psi(x), \psi(y)\} \in F$ whenever $\{x, y\} \in E)$ which is also onto whenever $G$ is finite;

(ii) if $G=H$ and $\varphi \mid S_{G}^{*}$ is the identity, then $\varphi$ is the identity.

Proof. By Lemma 5, $\varphi$ is one-to-one on $S_{G}^{*}$. Thus, by Lemma 6 (i) and (ii), $\psi: G \rightarrow H$ as given above is a well-defined one-to-one compatible mapping.

To complete the proof of (i), suppose $G$ is finite. Then, since $\Lambda\left(\left(\{x\}^{*}, 1\right)\right.$ : $x \in V)=(\varnothing, 0)$, it follows that

$$
\begin{aligned}
\bigwedge\left(\left(\{\psi(x)\}^{*}, 1\right): x \in V\right) & =\bigwedge\left((\{\psi(x)\}, 1)^{*}: x \in V\right)=\bigwedge\left((\varphi(\{x\}, 1))^{*}: x \in V\right) \\
& =\bigwedge\left(\varphi\left((\{x\}, 1)^{*}\right): x \in V\right)=\bigwedge\left(\varphi\left(\{x\}^{*}, 1\right): x \in V\right) \\
& =\varphi\left(\bigwedge\left(\left(\{x\}^{*}, 1\right): x \in V\right)\right)=(\varnothing, 0) .
\end{aligned}
$$

But as a meet of co-atoms of $S_{H}, \Lambda\left(\left(\{\psi(x)\}^{*}, 1\right): x \in V\right)$ can be $(\varnothing, 0)$ only if every co-atom is present, that is, $\psi(V) \supseteq W$.

Finally, if $G=H$ and $\varphi \uparrow S_{G}^{*}$ is the identity, then, by Lemma 6 (iii), $\varphi$ is the identity and (ii) holds.

\section{Proof of Theorem 1}

For $i<\aleph_{0}$, let $G(i)=(V(i), E(i))$ be the complete graph on $5+i$ elements, and let $S_{G(i)}$ be the associated pseudocomplemented semilattice. Clearly, for $i<\aleph_{0},|V(i)| \geq 5$ and $V(i) \subseteq \bigcup E(i)$.

Let $\left(U_{\alpha}: \alpha<2^{\aleph_{0}}\right)$ be a family of $2^{\aleph_{0}}$ distinct subsets of $\aleph_{0}$, and for each $\alpha<2^{\aleph_{0}}$ let $\mathbf{Q}_{\alpha}$ denote the quasivariety generated by the set $\left\{S_{G(i)}: i \in U_{\alpha}\right\}$.

Fix $\alpha, \beta<2^{\aleph_{0}}$ with $\alpha \neq \beta$. Without loss of generality, we may choose $m \in U_{\alpha} \backslash U_{\beta}$. If $S_{G(m)} \in \mathbf{Q}_{\beta}$, then $S_{G(m)} \in \mathbf{S P P}_{u}\left(S_{G(i)}: i \in U_{\beta}\right.$ ) (see Grätzer and Lakser [4]), and it follows (see Grätzer, Lakser, and Quackenbush [5]) from the fact that pseudocomplemented semilattices are locally finite (see Jones [7] and also Sankappanavar [10]) that $S_{G(m)} \in \mathbf{S P}\left(S_{G(i)}: i \in U_{\beta}\right)$. Given any $x \in$ $S_{G(m)}$ with $x \neq x^{* *}$, it follows that there exists $i \in U_{\beta}$ and a homomorphism $\varphi: S_{G(m)} \rightarrow S_{G(i)}$ such that $\varphi(x) \neq \varphi\left(x^{* *}\right)$. Hence the congruence induced by $\varphi$ fails to contain the Glivenko congruence. By Proposition 1 (i) it follows that $|G(m)|=|G(i)|$, which is absurd, and so the quasivarieties $\left(\mathbf{Q}_{\alpha}: \alpha<2^{\aleph_{0}}\right)$ are distinct. 


\section{Proof of Theorem 2}

To establish Theorem 2, we again choose suitable families of graphs, but this time with a little more care. By Hedrlin and Sichler [6], given a non-zero cardinal $\kappa$, there exists a family of graphs $(G(i)=(V(i), E(i)): i \in I)$ with the following properties: (i) each $G(i)$ is rigid, i.e., the only compatible mapping from $G(i)$ to itself is the identity; (ii) for $i \neq j$, there is no compatible mapping from $G(i)$ to $G(j)$; (iii) if $\kappa$ is infinite, then $|I|=2^{\kappa}$ and $|V(i)|=\kappa$ for $i \in I$; (iv) if $\kappa$ is finite, then $|I|=\kappa$ and $5 \leq|V(i)|=|V(j)|<\aleph_{0}$ for $i, j \in I$.

Consider $\left(S_{G(i)}: i \in I\right)$. By Proposition 1 (i), the absence of a compatible map from $G(i)$ to $G(j)$ implies that $S_{G(i)} \not S_{G(j)}$ for $i \neq j$.

For $i \in I$, let $\gamma_{i}$ be the Glivenko endomorphism of $S_{G(i)}$.

We show that for $i \in I, \operatorname{End}\left(S_{G(i)}\right)$ is isomorphic to the monoid $M \cup\{l\}$ where $M=\operatorname{End}\left(B_{G(i)}\right)$ and $\imath$ is an adjoined identity element, that is, $l \notin M$, $\imath^{2}=\imath$, and $\imath \psi=\psi l=\psi$ for all $\psi \in M$. Since $B_{G(i)} \cong B_{G(j)}$ for all $i, j \in I$, it will follow that $\operatorname{End}\left(S_{G(i)}\right) \cong \operatorname{End}\left(S_{G(j)}\right)$.

As $B_{G(i)} \cong S_{G(i)}^{*}$ it suffices to establish an embedding $T: \operatorname{End}\left(S_{G(i)}^{*}\right) \rightarrow$ $\operatorname{End}\left(S_{G(i)}\right)$ such that the image of $T$ consists precisely of all non-identity endomorphisms of $S_{G(i)}$. Define $T$ by $T(\alpha)=\alpha \gamma_{i}$ for all $\alpha \in \operatorname{End}\left(S_{G(i)}^{*}\right)$.

Since $\gamma_{i}$ is the identity on $S_{G(i)}^{*}, T$ is one-to-one and a homomorphism. Moreover, all $T(\alpha)$ are non-identity because $\gamma_{i}$ is not one-to-one.

To show that every non-identity $\varphi \in \operatorname{End}\left(S_{G(i)}\right)$ belongs to the image of $T$, it will suffice to show that each such $\varphi$ is equal to $\varphi^{\prime} \gamma_{i}$, where $\varphi^{\prime}$ denotes the restriction of $\varphi$ to $S_{G(i)}^{*}$. Equivalently, we need to show that the congruence induced by $\varphi$ contains the Glivenko congruence on $S_{G(i)}$. If such is not the case, then, by Proposition 1 (i), the rigidity of $G(i)$ implies that $\varphi^{\prime}$ is the identity. Whence, by Proposition 1 (ii), $\varphi$ is the identity in violation of the hypothesis.

\section{ACKNOWLEDGMENT}

We are pleased to acknowledge our debt to H. P. Sankappanavar for many stimulating and helpful discussions concerning pseudocomplemented semilattices. We are also grateful to J. Sichler for his comments and encouragement.

\section{REFERENCES}

1. M. E. Adams, Implicational classes of pseudocomplemented distributive lattices, J. London Math. Soc. 13 (1976), 381-384.

2. M. E. Adams, V. Koubek, and J. Sichler, Homomorphisms and endomorphisms in varieties of pseudocomplemented distributive lattices (with applications to Heyting algebras), Trans. Amer. Math. Soc. 285 (1984), 57-79.

3. G. Grätzer, General lattice theory, Birkhäuser Verlag, Basel and Stuttgart, 1978.

4. G. Grätzer and H. Lakser, $A$ note on the implicational class generated by a class of structures, Canad. Math. Bull. 16 (1973), 603-605. 
5. G. Grätzer, H. Lakser, and R. W. Quackenbush, On the lattice of quasivarieties of distributive lattices with pseudocomplementation, Acta Sci. Math. (Szeged) 42 (1980), 257-263.

6. Z. Hedrlin and J. Sichler, Any boundable binding category contains a proper class of mutually disjoint copies of itself, Algebra Universalis 1 (1971), 97-103.

7. G. T. Jones, Pseudocomplemented semilattices, Ph.D. Dissertation, U.C.L.A., 1972.

8. K. D. Magill, The semigroup of endomorphisms of a Boolean ring, Semigroup Forum 4 (1972), 411-416.

9. C. J. Maxson, On semigroups of Boolean ring endomorphisms, Semigroup Forum 4 (1972), 78-82.

10. H. P. Sankappanavar, Remarks on subdirectly irreducible pseudocomplemented semi-lattices and distributive pseudocomplemented lattices, Math. Japonica 25 (1980), 519-521.

11. B. M. Schein, Ordered sets, semilattices, distributive lattices, and Boolean algebras with homomorphic endomorphism semigroups, Fund. Math. 68 (1970), 31-50.

12. J. Schmid, Lee classes and sentences for pseudocomplemented semilattices, Algebra Universalis 25 (1988), 223-232.

13. A. Wroński, The number of quasivarieties of distributive lattices with pseudocomplementation, Polish Acad. Sci. Inst. Philos. Sociol. Sect. Logic 5 (1976), 115-121.

Department of Mathematics, State University of New York, New Paltz, New York 12561

Department of Mathematics, Vanderbilt University, Nashville, Tennessee 37235 